\title{
Ground Confluence
}

\author{
Richard Göbel \\ Fachbereich Informatik, Universität Kaiserslautem \\ Postfach 3049, D-6750 Kaiserslautern
}

\section{(1) Introduction}

The Knuth-Bendix Algorithm is a procedure for transforming a set of equations into a confluent term rewriting system. It has recently been applied in theories where only the congruence on ground terms of the equational theory is of interest, for example in the domain of inductive proofs (e.g. [MU 80], [HH 82], [KM 83], [JK 85]) or in the domain of program synthesis (e.g. [DE 85]). Although it is sufficient for these applications to generate term rewriting systems which are confluent on ground terms, the classical Knuth-Bendix Algorithm tries to generate a term rewriting system which is confluent on all terms. This often leads to cases where the classical Knuth-Bendix Algorithm generates an infinite system, even though the infinite system contains a finite ground confluent system. Unfortunately the ground confluence of a term rewriting system is in general undecidable [KN 87].

In this paper we will introduce a sufficient criterion for ground confluence which is stronger than the classical confluence test. This criterion allows us to prove the ground confluence of term rewriting systems where the classical Knuth-Bendix Algorithm does not terminate.

Our criterion for ground confluence seems to be the first general criterion for ground confluence which is stronger than the classical confluence test. On the other hand some work has been done in the context of inductive proofs to generate a ground confluent term rewriting system. In [GO 85] and [FR 86] not all critical pairs are generated for testing the ground confluence.

In [GO 85] a term rewriting system is split into three disjoint sets, a confluent set of rules (A) which are axioms for the inductive theory, a set of rules (I) which are inductive consequences of $A$ and a set containing all other rules $(\mathrm{O})$. The critical pairs are computed only from $\mathrm{A} \cup \mathrm{O}$ but they are reduced by all rules $(A \cup I \cup O)$. If the function symbols can be split into constructors and defined functions, then the number of critical pairs can be further reduced by considering only overlappings where variables are replaced by constructor terms.

In [FR 86] the term rewriting system is also split into axioms (A) and a set containing all other rules (O). Critical pairs between rules of $O$ are not considered and for a rule in $O$ only critical overlappings at a single position in the left hand side of the rule have to be considered. For this position a critical overlapping with a rule from $A$ has to exist for every (constructor-) ground instance of the rule. If no position in the rule satisfies this condition, then the confluence criterion can not be applied.

For both methods one needs special informations about the term rewriting system: 
- One has to to identify a subset of rules as axioms $\mathrm{A}$

- In [GO 85] one has to identify also a set of consequences I

- In [FR 86] one has to choose a position in the left hand side of a rule

If one is not able to identify a set of axioms in a set of rules $R$ which is smaller than $R$, then both methods can not be applied. This is the case for example in program synthesis where one starts with an unstructured set of equations. But also in the area of inductive proofs, one may get problems if the term rewriting system contains more than one complete set of axioms in a set of rules. In [GO 86] one can find an example with two complete sets of axioms, where the Knuth-Bendix Algorithm with the ground confluence criterion of [GO 85] or [FR 86] does not terminate if we choose the wrong set of axioms.

In [FR 86], one has to choose also positions in left hand sides of rules. Often, a wrong choice also causes the completion procedure to generate an infinite system.

In this paper we give a general ground confluence criterion for terminating term rewriting systems (section 3) which can be improved (section 4) for term rewriting systems with function symbols which occur in no irreducible ground term (convertible functions).

This general criterion has been implemented as part of a simple completion procedure. This implemetations allows also inductive proofs by consisteny with the KBA but does not require the choice of a set of axioms or the choice of a position in a rule as in [GO 85] and [FR 86]. With this implemetation we could not only prove the examples from [GO 85] and [FR 86] to be ground confluent but we also proved the ground confluence of systems where the methods in [GO 85] and [FR 86] fail, even though the conditions for the application of these methods were satisfied ([GO 86]).

\section{(2) Notation and Basic Definitions}

We assume familiarity of the reader with the basic proofs and results of the Knuth-Bendix Algorithm (e.g. [HU 77], [HO 80], [KB 70]), its extension for inductive proofs (e.g. [MU 80], [HH 82]) and the generalized Newman Lemma [WB 83].

We denote by VA the set of all variables and by $F S$ the set of all function symbols. TE(F,V) is the set of all terms constructed by variables from $V \subset V A$ and by function symbols from $F \subset F S$. A single term is denoted by $t$ or by $\alpha, \beta, \gamma$ or $\delta$ if it occurs in a rule or equation. Occurences in terms are denoted by $u, v$ and $w$. The symbol $\varepsilon$ denotes the top level occurence of a term. $O(t)$ is the set of all occurences of the term $t$ and $O^{\prime}(t)$ is the set of all non variable occurences of $t$. $V(t)$ returns all variables of the term $t$. Substitutions are denoted by $\sigma, \tau$ and $\varphi$.

A set of pairs of terms is denoted by $P$, if we consider this pairs as rules we will denote it by $R$ and if we consider these pairs as equations we will denote it by $\mathrm{E} . \alpha \rightarrow \beta$ and $\gamma \rightarrow \delta$ denote single rules and $\alpha=\beta$ and $\gamma=\delta$ denote single equations. For every term rewriting system $R$ in this paper we 
assume a $\mathrm{R}$-compatible term ordering $>(s \rightarrow \mathrm{R} t \Rightarrow \mathrm{s}>\mathrm{t})$, therefore we consider only terminating term rewriting systems. Further we assume that no left hand side of a rule consists of a single variable. A one step derivation with a rule in $P$ is denoted by $t \rightarrow P^{t^{\prime}}$ and $\rightarrow P$ is the symmetric closure of $\rightarrow \mathrm{p} . \stackrel{*}{\rightarrow} \mathrm{p}$ and ${ }^{*}-1 \mathrm{p}$ are the reflexive and transitive closures of $\rightarrow \mathrm{P}$ and $\mapsto \mathrm{p}$. Two terms $t_{1}$ and $t_{2}$ are subconnected in one step below a term $t\left(t_{1} \mapsto P, t t_{2}\right)$, if $t_{1} \mapsto p t_{2}, t>t_{1}$ and $t>$ $t_{2}$. The reflexive and transitive closure of $\mapsto P, t$ is denoted by ${ }^{*} t \mathrm{P}, \mathrm{t}$.

The set $I R R(R)$ contains all terms which are irreducible in $R$ and $I R R_{G}(R)$ contains all ground terms from IRR(R). Terms from IRR(R) are called to be in $R$ normal form. If thas a unique normal form in $R$, then this normal form is denoted by $t \downarrow R$

A term rewriting system is ground confluent, iff for all derivations $t_{1}$ and $t_{2}$ from a ground term $t$, there exists a term $t$ which is derivable from $t_{1}$ and $t_{2}$.

In this paper we will distinguish between critical overlappings $C O(R)$ and critical triples $C T(R)$ of a term rewriting system $\mathrm{R}$. These two sets are defined as follows:

\section{(2.1) Definition}

Let $\mathrm{R}$ be a term rewriting system. $\mathrm{CO}(\mathrm{R})$ denotes the set of all critical overiappings of $\mathrm{R}$ :

$$
\mathrm{CO}(\mathbf{R})=((\alpha \rightarrow \beta, \mathrm{u}, \gamma \rightarrow \delta) !
$$

$$
\left.\alpha \rightarrow \beta, \gamma \rightarrow \delta \in \mathrm{R} \wedge \mathrm{u} \in \mathrm{O}^{\prime}(\alpha) \wedge \exists \sigma, \tau: \sigma(\alpha) / \mathrm{u}=\tau(\gamma)\right)
$$

$\mathrm{CT}(\alpha \rightarrow \beta, \mathrm{u}, \gamma \rightarrow \delta)$ returns a critical triple for a critical overlapping from $\mathrm{CO}(\mathrm{R})$ :

$$
\mathrm{CT}(\alpha \rightarrow \beta, \mathrm{u}, \gamma \rightarrow \delta):=(\sigma(\alpha), \sigma(\alpha)[\mathrm{u} \leftarrow \sigma(\varphi(\delta))], \sigma(\beta))
$$

with: $-\varphi$ is a renaming substitution of $\gamma$

$-V(\varphi(\gamma)) \cap V(\alpha)=\varnothing$

- $\sigma$ is a most general unifier of $\alpha / \mathrm{u}$ and $\varphi(\gamma)$.

We denote the set of all critical triples of R by CTS(R).

The confluence and the subconnectedness of a critical triple are defined as follows:

\section{(2.2) Definition}

Let $R$ be a term rewriting system and $\left(t_{1}, t_{2}, t_{3}\right)$ be a triple of terms. Then:

- $\left(t_{1}, t_{2}, t_{3}\right)$ is confluent in $R$ iff $\exists t: t_{2} \stackrel{*}{\rightarrow} R t \wedge t_{3} \stackrel{*}{\rightarrow} R t$

$-\left(t_{1}, t_{2}, t_{3}\right)$ is subconnected in $R$ iff $t_{2} t^{*}-R, t_{1} t_{3}$.

\section{(3) A criterion for ground confluence}

In this section we introduce a sufficient criterion for ground confluence. We start with the observation that a term rewriting system is ground confluent iff every ground instance of a critical triple is 
confluent. Usually there are infinitely many ground instances of critical triples, therefore we have to find a finite sufficient test. For confluent critical triples the confluence of every ground instance of the triple is obviously satisfied, but all ground instances of other critical triples may be confluent even though the triple itself is not confluent. The key idea of our method is to generate a finite set $\mathrm{M}$ of instances for a critical triple $\left(t_{1}, t_{2}, t_{3}\right)$ which is not confluent. Then all ground instances of $\left(t_{1}, t_{2}, t_{3}\right)$ are confluent, if all triples in $M$ are confluent and the ground instances of $M$ contain all ground instances of $\left(t_{1}, t_{2}, t_{3}\right)$ (M covers $\left(t_{1}, t_{2}, t_{3}\right)$ ). Unfortunately this methods does not work for many interesting examples, because these finite sets $M$ do not exist for every critical triple which is not confluent. On the other hand we could find finite sets $\mathrm{M}$ covering these triples, where the sets $\mathrm{M}$ contain only subconnected critical triples. Then all ground instances of the critical triples are also subconnected. In fact the subconnectedness of every ground instance of a critical triple is sufficient for proving the ground confluence (theorem 3.2 basing on the result of [WB 83]).

Following this idea, the criterion for proving the ground confluence can be extended as follows:

- We create a finite set $M$ of instances for a critical triple $\left(t_{1}, t_{2}, t_{3}\right)$ if the critical triple is not confluent

- We prove that every triple $\left(t^{\prime}, t_{2}^{\prime}, t_{3}^{\prime}\right)$ from $M$ is subconnected

- The critical triple $\left(t_{1}, t_{2}, t_{3}\right)$ is ground subconnected if every ground instance of $\left(t_{1}, t_{2}, t_{3}\right)$ is also a ground instance of a triple in $\mathrm{M}$

We will now formalize these ideas. The proofs for this section and the next section can be found in [GO 86].

We will first give some notation:

\section{(3.1) Definition}

Let $\left(t_{1}, t_{2}, t_{3}\right)$ be a triple of terms and $M$ be a set of triples of terms.

- I(M) denotes the set of all ground instances of triples in $\mathrm{M}$ :

$$
\begin{aligned}
I(M):=\left\{\left(\sigma\left(t_{1}\right), \sigma\left(t_{2}\right), \sigma\left(t_{3}\right)\right) \mid\left(t_{1}, t_{2}, t_{3}\right)\right. & \in M \\
& \left.\wedge \forall x \in V\left(t_{1}\right) \cup V\left(t_{2}\right) \cup V\left(t_{3}\right): \sigma(x) \in T E(F S, \emptyset)\right\}
\end{aligned}
$$

- $\left(t_{1}, t_{2}, t_{3}\right)$ is ground confluent if all ground instances of $\left(t_{1}, t_{2}, t_{3}\right)$ are confluent: $\forall\left(\mathrm{t}^{\prime}, \mathrm{t}^{\prime}, \mathrm{t}^{\prime}\right) \in \mathrm{I}\left(\left\{\left(\mathrm{t}_{1}, \mathrm{t}_{2}, \mathrm{t}_{3}\right)\right\}\right) \exists \mathrm{t}^{\prime}: \mathrm{t}_{2}{ }_{2} \stackrel{*}{\rightarrow} \mathrm{R} \mathrm{t}^{\prime} \wedge \mathrm{t}_{3}^{\prime} \stackrel{*}{\rightarrow} \mathrm{R} \mathrm{t}^{\prime}$

- $\left(t_{1}, t_{2}, t_{3}\right)$ is ground subconnected if all ground instances of $\left(t_{1}, t_{2}, t_{3}\right)$ are subconnected:

$$
\forall\left(t^{\prime}{ }_{1}, t^{\prime}{ }_{2}, t^{\prime}{ }_{3}\right) \in I\left(\left\{\left(t_{1}, t_{2}, t_{3}\right)\right\}\right): t^{\prime}{ }_{2}{ }^{*}-1, t^{\prime} 1^{\prime}{ }_{3}^{\prime}
$$

The basis for this paper is the next theorem:

\section{(3.2) Theorem}

Let $R$ be a terminating term rewriting system. Then, $R$ is ground confluent iff every critical triple is ground subconnected:

$$
\forall\left(\mathrm{t}_{1}, \mathrm{t}_{2}, \mathrm{t}_{3}\right) \in \mathrm{I}(\mathrm{CTS}(\mathrm{R})): \mathrm{t}_{2}{ }^{*}{ }_{1} \mathrm{R}, \mathrm{t} 1 \mathrm{t}_{3}
$$


Every ground instance of a triple is subconnected if the triple is covered by a subconnected set of triples $M$ (definition 3.3, lemma 3.4):

\section{(3.3) Definition}

Let $M_{1}$ and $M_{2}$ be sets of triples of terms. $M_{1}$ covers $\mathbf{M}_{2}$ iff every ground instance of a triple in in $\mathrm{M}_{2}$ is also a ground instance of a triple in $\mathrm{M}_{1}$ :

$$
\mathrm{I}\left(\mathrm{M}_{2}\right) \subset \mathrm{I}\left(\mathrm{M}_{1}\right)
$$

\section{(3.4) Lemma}

Let $R$ be a term rewriting system, $\left(t_{1}, t_{2}, t_{3}\right)$ a triple of terms and $M$ be a set of triples of terms with:

$$
\forall\left(\mathrm{t}_{1}^{\prime}, \mathrm{t}^{\prime}, \mathrm{t}_{3}\right) \in \mathrm{I}(\mathrm{M}): \mathrm{t}_{2}^{\prime}{ }^{*}{ }^{*} \mathrm{R}, \mathrm{t}^{\prime} \mathrm{t}^{\prime}{ }_{3}
$$

Then, $\left(t_{1}, t_{2}, t_{3}\right)$ is ground subconnected if $M$ covers $\left(\left(t_{1}, t_{2}, t_{3}\right)\right)$.

Now we need a way to generate instances of critical triples and to prove that these instances are subconnected. The method in this paper bases on an extension of the confluence test given by [WB 83] and [KU 85].

For a critical triple which is not confluent we try to unify the left hand sides of rules with a subterm of the first component of the triple. Assume $\left(t_{1}, t_{2}, t_{3}\right)=C T(\alpha \rightarrow \beta, u, \gamma \rightarrow \delta)$ is a critical triple which is not confluent and $\gamma \rightarrow \delta^{\prime}$ is a rule where $\gamma^{\prime}$ is unifiable with $t_{1} / v$ by $\sigma$. Then the rules $\alpha \rightarrow \beta, \gamma \rightarrow \delta$ and $\gamma^{\prime} \rightarrow \delta^{\prime}$ can be applied at $\sigma\left(t_{1}\right):$

$$
\begin{aligned}
\sigma\left(\mathrm{t}_{1}\right) & \rightarrow \mathrm{R} \sigma\left(\mathrm{t}_{2}\right) & & \text { with } \gamma \rightarrow \delta \text { at } \mathrm{u} \\
& \rightarrow \mathrm{R} \sigma\left(\mathrm{t}_{1}\right)\left[\mathrm{v} \leftarrow \sigma\left(\delta^{\prime}\right)\right] & & \text { with } \gamma^{\prime} \rightarrow \delta^{\prime} \text { at } \mathrm{v} \\
& \rightarrow \mathrm{R} \sigma\left(\mathrm{t}_{3}\right) & & \text { with } \alpha \rightarrow \beta \text { at } \varepsilon
\end{aligned}
$$

The triple $\left(\sigma\left(t_{1}\right), \sigma\left(t_{2}\right), \sigma\left(t_{3}\right)\right)$ is subconnected if the triples $\left(\sigma\left(t_{1}\right), \sigma\left(t_{2}\right), \sigma\left(t_{1}\right)[v+\sigma(\delta)]\right)$ and $\left(\sigma\left(\mathrm{t}_{1}\right), \sigma\left(\mathrm{t}_{1}\right)[\mathrm{v} \leftarrow \sigma(\delta)], \sigma\left(\mathrm{t}_{3}\right)\right)$ are confluent:

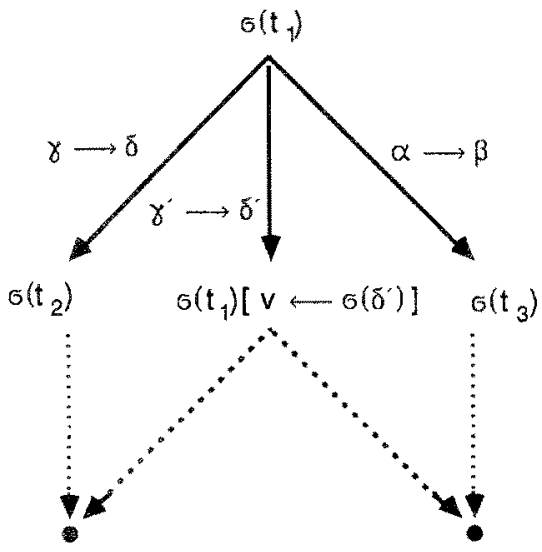

We try to prove the confluence of these triples by considering the positions where the rules $\gamma \rightarrow \delta$, $\gamma^{\prime} \rightarrow \delta^{\prime}$ are applied (case 1) for the triple $\left(\sigma\left(t_{1}\right), \sigma\left(t_{2}\right), \sigma\left(t_{1}\right)[v-\sigma(\delta)]\right)$ and the positions where 
the rules $\gamma^{\prime} \rightarrow \delta^{\prime}, \alpha \rightarrow \beta$ are applied (case 2$)$ for the triple $\left(\sigma\left(t_{1}\right), \sigma\left(t_{1}\right)\left[v \leftarrow \sigma\left(\delta^{\prime}\right)\right], \sigma\left(t_{3}\right)\right)$.

If two rules are applied at positions which do not critically overlap, then the derived terms can be reduced to a common term, otherwise we will check the critical overlappings between the rules.

We formalize this idea by introducing a new set $D C O(C, R)$ (double critical overlappings) of critical overlappings. $\operatorname{DCO}(\mathrm{C}, \mathrm{R})$ contains an element of the form $\left(\alpha \rightarrow \beta, u, \gamma \rightarrow \delta, v, \gamma^{\prime} \rightarrow \delta^{\prime}\right)$ if $(\alpha \rightarrow \beta, u, \gamma \rightarrow \delta)$ is an element from $C$ and a non variable subterm of the first component from $\left(t_{1}, t_{2}, t_{3}\right)=C T(\alpha \rightarrow \beta, u, \gamma \rightarrow \delta)$ is unifiable with the left hand side of a rule $\gamma^{\prime} \rightarrow \delta^{\prime}$ from $R$. We denote the instance of the triple CT $(\alpha \rightarrow \beta, u, \gamma \rightarrow \delta)$ by $\mathrm{DCT}\left(\alpha \rightarrow \beta, \mathrm{u}, \gamma \rightarrow \delta, v, \gamma^{\prime} \rightarrow \delta^{\prime}\right)$ (double critical triple). The set DCTS(C,R) contains a double critical triple for every element from $\operatorname{DCR}(\mathrm{C}, \mathrm{R})$.

\section{(3.5) Definition}

Let $R$ be a set of term rewriting rules and $C$ a subset of $C O(R)$. We define the sets DCO, DCT and DCTS on $R$ and $C$ as follows:

$$
\begin{aligned}
& \operatorname{DCO}(\mathrm{C}, \mathrm{R}):=\left\{\left(\alpha \rightarrow \beta, \mathrm{u}, \gamma \rightarrow \delta, \mathrm{v}, \gamma^{\prime} \rightarrow \delta^{\prime}\right) \mid(\alpha \rightarrow \beta, u, \gamma \rightarrow \delta) \in \mathrm{C} \wedge \gamma^{\prime} \rightarrow \delta^{\prime} \in \mathrm{R}\right. \\
& \wedge\left(\left(\mathrm{t}_{1}, \mathrm{t}_{2}, \mathrm{t}_{3}\right)=\mathrm{CT}(\alpha \rightarrow \beta, \mathrm{u}, \gamma \rightarrow \delta)\right. \\
& \left.\left.\Rightarrow \mathrm{v} \in \mathrm{O}^{\prime}\left(\mathrm{t}_{1}\right) \wedge \exists \sigma, \tau: \sigma\left(\mathrm{t}_{1}\right) / \mathrm{v}=\tau\left(\gamma^{\prime}\right)\right)\right\} \\
& \operatorname{DCT}\left(\alpha \rightarrow \beta, \mathrm{u}, \gamma \rightarrow \delta, \mathrm{v}, \gamma^{\prime} \rightarrow \delta^{\prime}\right):=\left(\sigma\left(\mathrm{t}_{1}\right), \sigma\left(\mathrm{t}_{2}\right), \sigma\left(\mathrm{t}_{3}\right)\right) \\
& \text { with: }\left(t_{1}, t_{2}, t_{3}\right)=\operatorname{CT}(\alpha \rightarrow \beta, u, \gamma \rightarrow \delta) \\
& \wedge \varphi^{\prime} \text { renaming substitution of } \gamma^{\prime} \text { and } V\left(\varphi^{\prime}(\gamma)\right) \cap V\left(t_{1}\right)=\varnothing \\
& \left.\wedge \sigma \text { is a most general unifier of } t_{1} / v \text { and } \varphi^{\prime}(\gamma)\right)
\end{aligned}
$$

$\operatorname{DCTS}(\mathrm{C}, \mathrm{R}):=\left\{\operatorname{DCT}\left(\alpha \rightarrow \beta, \mathrm{u}, \gamma \rightarrow \delta, \mathrm{v}, \gamma^{\prime} \rightarrow \delta^{\prime}\right)\right\}$

$$
\left.\left(\alpha \rightarrow \beta, \mathrm{u}, \gamma \rightarrow \delta, \mathrm{v}, \gamma^{\prime} \rightarrow \delta^{\prime}\right) \in \mathrm{DCO}(\mathrm{C}, \mathrm{R})\right\}
$$

In lemma 3.6 we give the criterion for the ground subconnectedness of a double critical triple $\operatorname{DCT}\left(\alpha \rightarrow \beta, \mathrm{u}, \gamma \rightarrow \delta, \mathrm{v}, \gamma^{\prime} \rightarrow \delta^{\prime}\right)$. If there is no critical overlapping between the rule $\gamma^{\prime} \rightarrow \delta^{\prime}$ and the rules $\alpha \rightarrow \beta$ and $\gamma \rightarrow \delta$ (the conditions $v \in O^{\prime}(\alpha), u=v . u^{\prime} \wedge u^{\prime} \in O^{\prime}\left(\gamma^{\prime}\right)$ and $v=u . v^{\prime} \wedge$ $\mathrm{v}^{\prime} \in \mathrm{O}^{\prime}(\gamma)$ are not satisfied), then the ground subconnectedness is immediately satisfied. Oherwise we have to check critical overlappings between the rule $\gamma^{\prime} \rightarrow \delta^{\prime}$ and the rules $\alpha \rightarrow \beta$ and $\gamma \rightarrow \delta$.

\section{(3.6) Lemma}

Let $\mathrm{R}$ be a term rewriting system and $\left(\alpha \rightarrow \beta, \mathrm{u}, \gamma \rightarrow \delta, \mathrm{v}, \gamma^{\prime} \rightarrow \delta^{\prime}\right)$ be from $\mathrm{DCO}(\mathrm{CO}(\mathrm{R}), \mathrm{R})$. The triple $\mathrm{DCT}\left(\alpha \rightarrow \beta, u, \gamma \rightarrow \delta, v, \gamma \rightarrow \delta^{\prime}\right)$ is ground subconnected, if:

$$
\begin{array}{ll} 
& \left(v \in O^{\prime}(\alpha) \Rightarrow \operatorname{CT}\left(\alpha \rightarrow \beta, v, \gamma^{\prime} \rightarrow \delta^{\prime}\right) \text { is confluent }\right) \\
\wedge \quad\left(u=v . u^{\prime} \wedge u^{\prime} \in O^{\prime}\left(\gamma^{\prime}\right) \Rightarrow \operatorname{CT}\left(\gamma^{\prime} \rightarrow \delta^{\prime}, u^{\prime}, \gamma \rightarrow \delta\right) \text { is confluent }\right) \\
\wedge \quad\left(v=u . v^{\prime} \wedge v^{\prime} \in O^{\prime}(\gamma) \Rightarrow \operatorname{CT}\left(\gamma \rightarrow \delta, v^{\prime}, \gamma^{\prime} \rightarrow \delta^{\prime}\right) \text { is confluent }\right)
\end{array}
$$

Basing on these definitions and Lemmata, we can give a criterion for ground confluence: 


\section{Ground Confluence Criterion}

(1) Let: CONFL-CO: $=((\alpha \rightarrow \beta, u, \gamma \rightarrow \delta) \mid(\alpha \rightarrow \beta, u, \gamma \rightarrow \delta) \in \mathrm{CO}(\mathrm{R})$

$$
\begin{gathered}
\text { and } \mathrm{CT}(\alpha \rightarrow \beta, \mathrm{u}, \gamma \rightarrow \delta) \text { is confluent }\} \\
\text { NOT-CONFL-CO }:=\{(\alpha \rightarrow \beta, \mathrm{u}, \gamma \rightarrow \delta) \mid(\alpha \rightarrow \beta, \mathrm{u}, \gamma \rightarrow \delta) \in \mathrm{CO}(\mathrm{R}) \\
\text { and } \mathrm{CT}(\alpha \rightarrow \beta, \mathrm{u}, \gamma \rightarrow \delta) \text { is not confluent }\}
\end{gathered}
$$

(2) Let: CONNECTED-DCO

$$
\begin{aligned}
:=\{(\alpha \rightarrow \beta, & \left., \gamma \rightarrow \delta, v, \gamma \rightarrow \delta^{\prime}\right) \mid \\
& \left.\left(\alpha \rightarrow \beta, u, \gamma \rightarrow \delta, v, \gamma^{\prime} \rightarrow \delta\right) \in \text { DCO(NOT-CONFL-CO, R }\right) \\
& \wedge\left(v \in O^{\prime}(\alpha) \Rightarrow\left(\alpha \rightarrow \beta, v, \gamma^{\prime} \rightarrow \delta^{\prime}\right) \in \text { CONFL-CO }\right) \\
& \wedge\left(u=v, u^{\prime} \wedge u^{\prime} \in O^{\prime}\left(\gamma^{\prime}\right) \Rightarrow\left(\gamma^{\prime} \rightarrow \delta^{\prime}, u^{\prime}, \gamma \rightarrow \delta\right) \in \text { CONFLCO }\right) \\
& \left.\wedge\left(v=u . v^{\prime} \wedge v^{\prime} \in O^{\prime}(\gamma) \Rightarrow\left(\gamma \rightarrow \delta, v^{\prime}, \gamma^{\prime} \rightarrow \delta\right) \in \text { CONFL-CO }\right)\right\}
\end{aligned}
$$

(3) Then $R$ is ground confluent, if CONNECTED-DCO $\cup$ CONFL-CO covers NOT-CONFL-CO

For the correctness of this criterion, we have to prove that every critical triple of $\mathrm{R}$ is ground subconnected (Theorem 3.2). If a triple is confluent, then it is also ground subconnected. Otherwise we consider a set of subconnected triples CONNECTED-DCO (Lemma 3.6). Then, the critical triple is subconnected if it is covered by the set of subconnected triples CONNECTED-DCO $U$ CONFL-CO (Lemma 3.4).

To complete this confluence criterion, we need a method for proving the coveredness property. With the test of Kounalis [KO 85] for example we can prove the coveredness of a set of terms by another set of terms. This test can be extended to triples of terms by introducing a new ternary operator $f$ and applying it to every triple. Then, we check the coveredness of a set of triples $M_{1}$ by a set of triples $\mathrm{M}_{2}$ as follows:

(1) Transform the triples in $M_{1}$ and $M_{2}$ into terms:

$$
\begin{aligned}
& M_{1}^{*}:=\left\{f\left(t_{1}, t_{2}, t_{3}\right) \mid\left(t_{1}, t_{2}, t_{3}\right) \in M_{1}\right\} \\
& M_{2}^{\prime}:=\left\{f\left(t_{1}, t_{2}, t_{3}\right) \mid\left(t_{1}, t_{2}, t_{3}\right) \in M_{2}\right\}
\end{aligned}
$$

(2) $M_{1}$ is covered by $M_{2}$ iff $M_{1}^{\prime}$ is covered by $M_{2}^{\prime}$ 


\section{(4) An extension to convertible functions}

In this section we introduce a weaker rewrite relation $\rightarrow R$. For this relation a rule may only be applied if the variables on the left hand side of the rule are replaced by normal forms (definition 4.1). The relation $\rightarrow R$ is ground confluent iff the relation $\rightarrow R$ is ground confluent (theorem 4.2), therefore it is sufficient to prove the ground confluence of $\rightarrow R$. We now assume that some function symbols do not occur in ground term normal forms (convertible functions). Then a rule can not be applied to a ground term if a variable of the rule is replaced by a term containing at least one of the convertible function symbols. Therefore we consider only overlappings where variables are replaced by terms without convertible function symbols (theorem 4.5 ). Further we consider instances sets $\mathrm{M}$ of critical triples covering only ground instances of triples where variables are replaced by terms without convertible functions (lemma 4.7).

\section{(4.1) Definition}

Let $R$ be a term rewriting system. We define the relations $\rightarrow R, \Vdash-\| R$ and $\|--\| R, t$ as follows:

$$
\begin{aligned}
& -\mathbf{t}_{\mathbf{1}} \longrightarrow \mathbf{R} \mathbf{t}_{\mathbf{2}} \Leftrightarrow: \exists \alpha \rightarrow \beta \in \mathrm{R}: \exists \sigma \exists \mathrm{u} \in \mathrm{O}(\mathrm{t}): \mathrm{t}_{\mathrm{l}} / \mathrm{u}=\sigma(\alpha) \\
& \wedge(\forall x \in V(\alpha): \sigma(x) \in \operatorname{IRR}(R)) \\
& \wedge t_{2}=t_{1}[u \leftarrow \sigma(\beta)] \\
& -t_{1} \Vdash R t_{2} \quad \Leftrightarrow: t_{1} \rightarrow R t_{2} \vee t_{2} \rightarrow R t_{1} \\
& -t_{1} \mid \mapsto-R, t t_{2} \Leftrightarrow: t_{1} \Vdash-\| t_{2} \wedge t>t_{1} \wedge t>t_{2}
\end{aligned}
$$

We denote by $\stackrel{*}{\rightarrow}$ the reflexive and transitive closure of $\rightarrow$ and by $\Perp^{*}$ the reflexive and transitive closure of $\Vdash-\|$.

The relations $\rightarrow R$ and $\rightarrow R$ have the same normal forms, their symmetric, transitive and reflexive closures are equivalent and $\rightarrow R$ is confluent iff $\rightarrow R$ is confluent:

\section{(4.2) Theorem}

Let $R$ be a term rewriting system. Then:

- $\forall \mathrm{t}: \mathrm{t}$ irreducible by $\rightarrow \mathrm{R} \Leftrightarrow \mathrm{t}$ irreducible by $\rightarrow \mathrm{R}$

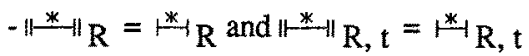

$-\rightarrow R$ is (ground-) confluent iff $\rightarrow R$ is (ground-) confluent.

Note that these results for $\rightarrow R$ are not restricted to ground terms, but only the restriction to ground terms allows us to restrict the replacement of variables to terms without convertible functions.

We now modify the criterion given in section 2 for the relation $\rightarrow R$ by considering only those function symbols $\mathrm{F}$ which are not convertible for the computation of critical overlappings and for the coveredness criterion of a critical triple.

In definition 4.3 we restrict the critical overlappings to cases where variables in rules can be replaced by terms without convertible functions: 


\section{(4.3)Definition}

Let $R$ be a term rewriting system and F $\subset$ FS. We define the set of critical overlappings $C O_{F}(R)$ and the set of critical triples $\operatorname{CTS}_{F}(R)$ as follows:

$$
\begin{aligned}
& \mathrm{CO}_{\mathrm{F}}(\mathrm{R}):=\left\{(\alpha \rightarrow \beta, \mathrm{u}, \gamma \rightarrow \delta) \mid \alpha \rightarrow \beta, \gamma \rightarrow \delta \in \mathrm{R} \wedge \mathrm{u} \in \mathrm{O}^{\prime}(\alpha)\right. \\
& \wedge \exists \sigma, \tau: \quad \sigma(\alpha) / \mathrm{u}=\tau(\gamma) \\
& \wedge \forall \mathrm{x} \in \mathrm{V}(\alpha): \sigma(\mathrm{x}) \in \mathrm{TE}(\mathrm{F}, \mathrm{VA}) \\
&\wedge \forall \mathrm{x} \in \mathrm{V}(\gamma): \tau(\mathrm{x}) \in \mathrm{TE}(\mathrm{F}, \mathrm{VA})\}
\end{aligned}
$$

$\operatorname{CTS}_{\mathrm{F}}(\mathrm{R}):=\left\{\mathrm{CT}(\alpha \rightarrow \beta, \mathrm{u}, \gamma \rightarrow \delta) \mid(\alpha \rightarrow \beta, u, \gamma \rightarrow \delta) \in \mathrm{CO}_{\mathrm{F}}(\mathrm{R})\right\}$

Note, that $C O(R)$ is equal to $C_{F S}(R)$ and $C T S(R)$ is equal to $\operatorname{CTS}_{F S}(R)$.

Instead of considering all ground instances of triples, we consider only instances where variables are replaced by ground terms without convertible function symbols:

(4.4) Definition (modified version of definition 3.1)

Let $\left(t_{1}, t_{2}, t_{3}\right)$ be a triple of terms, $F \subset F S$ a set of function symbols and $M$ be a set of triples of terms.

- $I_{F}(M)$ denotes the set of all ground instances in $F$ of triples in $M$ :

$$
\begin{aligned}
\mathbf{I}_{\mathbf{F}}(\mathbf{M}):=\left\{\left(\sigma\left(\mathrm{t}_{1}\right), \sigma\left(\mathrm{t}_{2}\right), \sigma\left(\mathrm{t}_{3}\right)\right) \mid\left(\mathrm{t}_{1}, \mathrm{t}_{2}, \mathrm{t}_{3}\right) \in \mathrm{M}\right. \\
\left.\qquad \forall \mathrm{x} \in \mathrm{V}\left(\mathrm{t}_{1}\right) \cup \mathrm{V}\left(\mathrm{t}_{2}\right) \cup \mathrm{V}\left(\mathrm{t}_{3}\right): \sigma(\mathrm{x}) \in \mathrm{TE}(\mathrm{F}, \varnothing)\right\}
\end{aligned}
$$

- $\left(t_{1}, t_{2}, t_{3}\right)$ is ground confluent in $F$ if all ground instances in $F$ of $\left(t_{1}, t_{2}, t_{3}\right)$ are confluent:

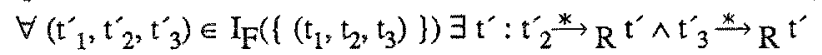

- $\left(t_{1}, t_{2}, t_{3}\right)$ is ground subconnected in $F$ if all ground instances in $F$ of $\left(t_{1}, t_{2}, t_{3}\right)$ are subconnected:

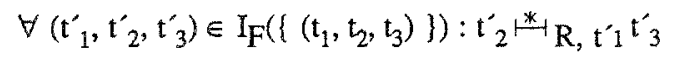

A term rewriting system is ground confluent if all ground instances in $F$ of $\operatorname{CTS}_{F}(R)$ are subconnected and FSY contains only convertible function symbols:

(4.5) Theorem (modified version of theorem 3.2)

Let $R$ be a terminating term rewriting system, $F \subset F S$ a set of function symbols and FS $F$ contains only convertible function symbols. Then, $R$ is ground confluent iff:

$$
\forall\left(t_{1}, t_{2}, t_{3}\right) \in I_{F}\left(\operatorname{CTS}_{F}(R)\right): t_{2} *_{1} \cdot R, t_{1} t_{3}
$$

The definition of the coveredness property can also be extended for convertible functions:

\section{(4.6) Definition}

(modified version of definition 3.3)

Let $M_{1}$ and $M_{2}$ be sets of triples of terms and $F \subset F S$ a set of function symbols. $M_{1}$ covers $M_{2}$ in iff:

$$
\mathrm{IF}_{\mathrm{F}}\left(\mathrm{M}_{2}\right) \subset \mathrm{I}_{\mathrm{F}}\left(\mathrm{M}_{1}\right)
$$


Every ground instance in $F$ of a critical triple is subconnected if it is covered in $F$ by a subconnected set of triples $\mathrm{M}$ :

\section{(4.7) Lemma (modified version of lemma 3.4)}

Let $R$ be a term rewriting system, $F \in F S$ a set of function symbols, $\left(t_{1}, t_{2}, t_{3}\right)$ a triple of terms and $M$ be a set of triples of terms with:

$$
\forall\left(\mathrm{t}^{\prime}{ }_{1}, \mathrm{t}^{\prime}{ }_{2}, \mathrm{t}^{\prime}\right) \in \mathrm{I}_{\mathrm{F}}(\mathrm{M}): \mathrm{t}_{2}{ }^{\prime}{ }^{*} \mathrm{R}, \mathrm{t}^{\prime} \mathrm{t}^{\prime}{ }_{3}
$$

Then, $\left(t_{1}, t_{2}, t_{3}\right)$ is ground subconnected in $F$ if $M$ covers $\left\{\left(t_{1}^{\prime}, t_{2}^{\prime}, t^{\prime}\right)\right\}$.

Now, we can also give a modified criterion for ground confluence:

Ground Confluence Criterion

(1) Let: CONFL-CO $:=\left\{(\alpha \rightarrow \beta, u, \gamma \rightarrow \delta) \mid(\alpha \rightarrow \beta, u, \gamma \rightarrow \delta) \in \mathrm{CO}_{\mathrm{FS}}(\mathrm{R})\right.$

$$
\begin{gathered}
\text { and CT }(\alpha \rightarrow \beta, u, \gamma \rightarrow \delta) \text { is confluent }\} \\
\text { NOT-CONFL-CO }:=\left\{(\alpha \rightarrow \beta, u, \gamma \rightarrow \delta) \mid(\alpha \rightarrow \beta, u, \gamma \rightarrow \delta) \in \mathrm{CO}_{\mathrm{F}}(\mathrm{R})\right. \\
\text { and CT }(\alpha \rightarrow \beta, u, \gamma \rightarrow \delta) \text { is not confluent }\}
\end{gathered}
$$

(2) Let: CONNECTED-DCO

$$
\begin{aligned}
:= & \left\{\left(\alpha \rightarrow \beta, u, \gamma \rightarrow \delta, v, \gamma^{\prime} \rightarrow \delta^{\prime}\right) \mid\right. \\
& \left.\left(\alpha \rightarrow \beta, u, \gamma \rightarrow \delta, v, \gamma^{\prime} \rightarrow \delta^{\prime}\right) \in \text { DCO(NOT-CONFL-CO, R }\right) \\
& \wedge\left(v \in O^{\prime}(\alpha) \Rightarrow\left(\alpha \rightarrow \beta, v, \gamma^{\prime} \rightarrow \delta^{\prime}\right) \in \text { CONFL-CO }\right) \\
& \wedge\left(u=v . u^{\prime} \wedge u^{\prime} \in O^{\prime}\left(\gamma^{\prime}\right) \Rightarrow\left(\gamma^{\prime} \rightarrow \delta^{\prime}, u^{\prime}, \gamma \rightarrow \delta\right) \in \text { CONFL-CO }\right) \\
& \left.\wedge\left(v=u . v^{\prime} \wedge v^{\prime} \in O^{\prime}(\gamma) \Rightarrow\left(\gamma \rightarrow \delta, v^{\prime}, \gamma \rightarrow \delta\right) \in \text { CONFL-CO }\right)\right\}
\end{aligned}
$$

(3) Then $\mathrm{R}$ is ground confluent, if CONNECTED-DCO $\cup$ CONFL-CO covers NOT-CONFL-CO in F

Note, that we consider all confluent critical triples from $\mathrm{CO}_{\mathrm{FS}}(\mathrm{R})$ because this may help us to prove more triples in DCO(NOT-CONFL-CO, R) to be confluent than to consider only confluent critical triples from $\mathrm{CO}_{\mathrm{F}}(\mathrm{R})$.

The coveredness test of Kounalis [KO 85] has not to be modified, because it allows us to distinguish constructors (F) and defined function symbols (FSVF), and to prove the coveredness of a set terms only for constructor ground instances.

\section{(5) Conclusion}

The ground confluence criterion developed in this paper was implemented as part of simple completion procedure. This implementation allowed us to prove the ground confluence of many systems where the classical Knuth-Bendix generates an infinite system. We can prove also the 
ground confluence of every confluent and terminating term rewriting system because the criterion contains completely the classical confluence test. If we apply our criterion for inductive proofs, than the ground confluence can be proven without splitting the term rewriting system into axioms and other rules and without choosing position in rules, which was necessary in [GO 85] or [FR 86]. In fact, we could prove more systems to be ground confluent than with the methods given in [GO 85] and [FR 86], but it is not obvious that our method is a stronger criterion for any term rewriting system.

\section{Appendix Example}

Here, we show by an example how our ground confluence test works. This example has been considered in [FR 86] and could be proven to be ground confluent, even though the classical Knuth-Bendix Algorithm generates an infinite set of rules. Other examples can be found in [GO 86].

Consider the following set of rules:
(1) add $(0, y) \rightarrow y$
(2) $\operatorname{add}(s(x), y) \rightarrow s(\operatorname{add}(x, y))$
(3) $\operatorname{add}(\mathrm{u}, \operatorname{add}(\mathrm{v}, \mathrm{w})) \rightarrow \operatorname{add}(\operatorname{add}(\mathrm{u}, \mathrm{v}), \mathrm{w})$

For this Term rewriting system add is convertible.

We get the following critical overlappings and critical triples:

(rule 3, 2, rule 2) : ( add(u,add(s(x),y)), add(u,s(add(x,y))), add(add(u,s(x)),y))

(rule 3, $\varepsilon$, rule 2) : (add(s(x),add(v,w)), s(add(x,add(v,w))), add(add(s(x),v),w))

(rule 3, 2, rule 1) : ( add(u,add $(0, y))$, add(u,y), add(add $(u, 0), y)$ )

(rule $3, \varepsilon$, rule 1$):(\operatorname{add}(0, \operatorname{add}(v, w))$, add $(v, w)$, add $(\operatorname{add}(0, v), w)$ )

(nule 3, 2, rule 3) : (add(x,add(y,add $(\mathrm{z}, \mathrm{u})))$, add( $(\mathrm{x}, \operatorname{add}(\operatorname{add}(\mathrm{y}, \mathrm{z}), \mathrm{u}))$, add(add $(\mathrm{x}, \mathrm{y}), \operatorname{add}(\mathrm{z}, \mathrm{u})))$

Now, the critical triples of the second critical overlapping (rule $3, \varepsilon$, rule 2), the fourth critical overlapping (rule 3 , $\varepsilon$, rule 1) and the fifth critical overlapping (rule 3,2, rule 3) are confluent. The other critical triples:

(rule 3,2 , rule 2$):($ add $(u, a d d(s(x), y))$, add(u,s(add $(x, y)))$, add(add $(u, s(x)), y))$

(rule 3,2, nile 1$)$ : ( add $(u, \operatorname{add}(0, y))$, add( $u, y)$, add(add $(u, 0), y)$ )

are not confluent but belong to $\mathrm{CO}_{\mathrm{F}}(\mathrm{R})$ therefore we generate instances by unifying left hand sides of rules with their first elements:

(rule 3,2 , rule $2, \varepsilon$, rule 2 ) :

$(\operatorname{add}(s(u), \operatorname{add}(s(x), y)), \operatorname{add}(s(u), s(\operatorname{add}(x, y))), \operatorname{add}(\operatorname{add}(s(u), s(x)), y))$

(nule 3,2 , rule $2, \varepsilon$, rule 1 ) :

$(\operatorname{add}(0, \operatorname{add}(s(x), y)), \operatorname{add}(0, s(\operatorname{add}(x, y))), \operatorname{add}(\operatorname{add}(0, s(x)), y))$

(rule 3,2 , rule $1, \mathrm{E}$, rule 2 ) :

$(\operatorname{add}(s(u), \operatorname{add}(0, y)), \operatorname{add}(s(u), y), \operatorname{add}(\operatorname{add}(s(u), 0), y))$

(rule 3,2 , rule $1, \varepsilon$, rule 1 ) :

$(\operatorname{add}(0, \operatorname{add}(0, y)), \operatorname{add}(0, y), \operatorname{add}(\operatorname{add}(0,0), y))$

Now, we can prove that all these triples are subconnected. Consider for example the triple of the overlapping (rule 3,2 , rule $2, \varepsilon$, rule 2 ):

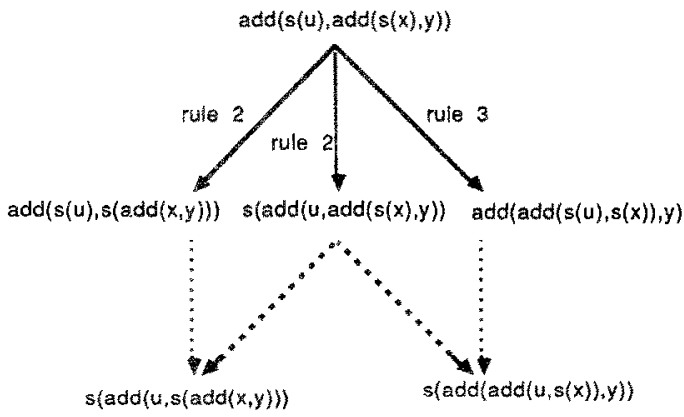


This triple is subconnected because there is no critical overlapping between the two positions where rule 2 is applied and the critical overlapping between rule 3 and rule 2 is confluent. The triple is not confluent because we are not able to derive a common term from $\operatorname{add}(s(u), s(\operatorname{add}(x, y)))$ and add $(\operatorname{add}(s(u), s(x)), y))$.

\section{References}

[DE 85] Dershowitz N.: Synthesis by Completion

Proc. 9th LCAI, Los Angeles 1985

[FR 86] Fribourg L.: A Strong Restriction of the Inductive Completion Procedure Proc. ICALP 86, 1986

[GO 85] Göbel R.: A Specialized Knuth-Bendix Algorithm for Inductive Proofs

Proc. Combinatorial Algorithms in Algebraic Structures, Universität Kaiserslautern 1985

[GO 86] Gobel R: Ground Confluence

SEKI-Report SR-86-18, Universität Kaiserslautern 1986

[HH 82] Huet G., Hullot J.: Proofs by Induction in Equational Theories with Constructors

Journal of the Association for Computing Machinery 25(2), p. 239-266, 1982

[HO 80] Huet, G:, Oppen D.: Equations and Rewrite Rules: A Survey

Technical Report CSL - 111, SRI International 1980

[HU 77] Huet, G.: Confluent Reductions: Abstract Properties and Applications to Term Rewriting Systems $18 \mathrm{th}$ IEEE Symposium on foundations of Computer Science, p. $30-45,1977$

[JK 85] Jouannaud J.-P., Kounalis E.: Proofs by Induction in Equational Theories without Constructors CRIN, University de Nancy, Nancy 1985

[KB 70] Knuth D., Bendix P.: Simple Word Problems in Universal Algebras Computational Problems in Abstract Algebra, Ed. Leech I., Pergamon Press, p. 263 - 297, 1970

[KN 87] Kapur D.,Narendran P., Otto F.: On ground confluence of term rewriting systems submitted for publication, 1987

[KM 83] Kapur D., Musser D. R.: Proofs by Consistency

Proc. of an NSF Workshop on the Rewrite Rule Laboratory Sept. 83

General Electric R \& D Center Report GEN84008, Schenectady 1984

[KO 85] Kounalis E.: Completeness in Data Type Specifications

Proc. EUROCAL 85, Springer Lecture Notes, Linz 1985

[KU 85] Küchlin W.: A Confluence Criterion Based on the Generalised Newman Lemma Proc. EUROCAL 85, Springer Lecture Notes, Linz 1985

[MU 80] Musser D.: On Proving Inductive Properies of Abstract Data Types 7th Annual ACM Symposium on Principles of Programming Languages, Las Vegas 1980

[WB 83] Winkler F., Buchberger B:

A Criterion for Eliminating Unnecessary Reductions in the Knuth-Bendix Algorithm Colloqium on Algebra, Combinatorics and Logic in Computer Science, Györ (Hungary) 1983 